\title{
Transcriptional responses to glucose in Saccharomyces cerevisiae strains lacking a functional protein kinase $\mathrm{A}$
}

\author{
Daniela Livas ${ }^{1}$, Marinka JH Almering², Jean-Marc Daran², Jack T Pronk² and Juana M Gancedo ${ }^{\text {1* }}$
}

\begin{abstract}
Background: The pattern of gene transcripts in the yeast Saccharomyces cerevisiae is strongly affected by the presence of glucose. An increased activity of protein kinase A (PKA), triggered by a rise in the intracellular concentration of CAMP, can account for many of the effects of glucose on transcription. In S. cerevisiae three genes, TPK1, TPK2, and TPK3, encode catalytic subunits of PKA. The lack of viability of tpk1 tpk2 tpk3 triple mutants may be suppressed by mutations such as yak 1 or msn2/msn4. To investigate the requirement for PKA in glucose control of gene expression, we have compared the effects of glucose on global transcription in a wild-type strain and in two strains devoid of PKA activity, tpk1 tpk2 tpk3 yak1 and tpk1 tpk2 tpk3 msn2 msn4.
\end{abstract}

Results: We have identified different classes of genes that can be induced -or repressed- by glucose in the absence of PKA. Representative examples are genes required for glucose utilization and genes involved in the metabolism of other carbon sources, respectively. Among the genes responding to glucose in strains devoid of PKA some are also controlled by a redundant signalling pathway involving PKA activation, while others are not affected when PKA is activated through an increase in CAMP concentration. On the other hand, among genes that do not respond to glucose in the absence of PKA, some give a full response to increased CAMP levels, even in the absence of glucose, while others appear to require the cooperation of different signalling pathways. We show also that, for a number of genes controlled by glucose through a PKA-dependent pathway, the changes in mRNA levels are transient. We found that, in cells grown in gluconeogenic conditions, expression of a small number of genes, mainly connected with the response to stress, is reduced in the strains lacking PKA.

Conclusions: In S. cerevisiae, the transcriptional responses to glucose are triggered by a variety of pathways, alone or in combination, in which PKA is often involved. Redundant signalling pathways confer a greater robustness to the response to glucose, while cooperative pathways provide a greater flexibility.

\section{Background}

Adaptation to changing environmental conditions is of capital importance for the survival of organisms and their successful establishment in new ecological niches. To enable adaptation, sophisticated networks of signalling that govern growth and development have been selected in all living systems. In the model yeast Saccharomyces cerevisiae, availability of glucose or other easily fermentable sugars elicits a drastic rearrangement of metabolism and multiple changes in its transcriptome. An immediate

\footnotetext{
* Correspondence: jmgancedo@iib.uam.es

'Department of Metabolism and Cell Signalling, Instituto de Investigaciones Biomédicas Alberto Sols, CSIC-UAM, Arturo Duperier 4, 28029 Madrid, Spain Full list of author information is available at the end of the article
}

consequence of the exposure to glucose is a rise in the concentration of internal cAMP $[1,2]$, and it is well established that changes in cAMP levels affect the transcription rate of a large number of genes in S. cerevisiae [3-5]. The increase in cAMP levels is mainly due to the activation of the plasma membrane-bound adenylate cyclase mediated by different $\mathrm{G}$ proteins, Ras1, Ras2 and Gpa2 [6,7]. The GDP-GTP exchange in the Ras1 or Ras2 proteins is mediated by Cdc25 [8], which is activated by glucose by an, as yet, unknown mechanism [9]. Activation of Gpa2, in turn, results from the binding of glucose to the transmembrane protein Gpr1 [10-12]. Bcy1, the regulatory subunit of the cAMP-dependent protein kinase (PKA), when binding cAMP, dissociates from the

\section{Biomed Central}


catalytic subunits and thereby activates them. In a yeast strain harbouring a $R A S 2^{\text {Val19 }}$ gene, encoding a constitutively active form of Ras2, under the control of the GAL1 promoter, an increase in internal cAMP levels may be achieved in the absence of glucose through the addition of galactose. Even when galactose metabolism in such a strain is blocked by disruption of the GAL1 gene (encoding galactokinase), the increase in cAMP levels that follows galactose addition causes many of the effects on transcription that are elicited by glucose [5]. The same authors also report that in a bcyl strain, where PKA activity is independent of cAMP levels, transcription of many genes still responds to the presence of glucose. This last observation suggests that there are additional, and possibly redundant, glucose signalling pathway(s) independent of changes in PKA activity triggered by cAMP [5]. This does not, however, discard the possibility that the alternative pathway(s) require a basal activity of PKA, even if they do not depend on its activation by cAMP.

The goal of the present study was to investigate the occurrence of PKA-independent glucose signalling in $S$. cerevisiae. To this end, we have used global transcription analysis to study the effects of glucose on yeast strains completely devoid of PKA activity. In $S$. cerevisiae three genes TPK1, TPK2, and TPK3 encode catalytic subunits of PKA. While strains expressing only one of these genes grow normally, a triple null mutant (tpk1 tpk2 tpk3) is not viable [13]. Characterization of different mutations able to suppress the growth defect of the triple mutant [14-16] has enabled identification of the crucial function of PKA. As shown in Figure 1, PKA is needed to counteract the negative effect of the protein kinase Yak1 on yeast growth $[17,18]$. In the presence of PKA the protein kinase Rim15 [15] and the transcription factors Msn2 and Msn4 [19] can be phosphorylated and exported to the cytoplasm. As a consequence, transcription of Msn2/ Msn4 activated genes, among them YAK1 [16], is reduced, Yak1 levels remain low and growth is not hindered. In the absence of PKA, Rim15 remains in the nucleus where it can activate Msn2/Msn4 [20] that turn on $Y A K 1$ transcription. Yak1 then blocks growth by mechanisms that may include the phosphorylation and activation of Msn2/Msn4 [21]. This explains why strains lacking Rim15, Msn2/Msn4 or Yak1 no longer require PKA for growth. In this work we have used two isogenic strains lacking PKA and carrying the suppressor mutations msn 2 msn4 or yak1. Two different suppressor mutants were used with the aim to enable a dissection of effects of the lack of PKA and effects of the suppressor mutations themselves.

We found that glucose induction of genes related with glucose metabolism or glucose repression of genes involved in the utilization of carbon sources alternative to glucose can proceed in the absence of PKA. We noted, however, that genes related with similar types of processes, such as biosynthesis of amino acids or transport of drugs, may respond differently to the lack of PKA.

\section{Results}

\section{Genes induced by glucose}

When ethanol-grown cultures of the TPK1 TPK2 TPK3 reference strain were exposed to glucose over 700
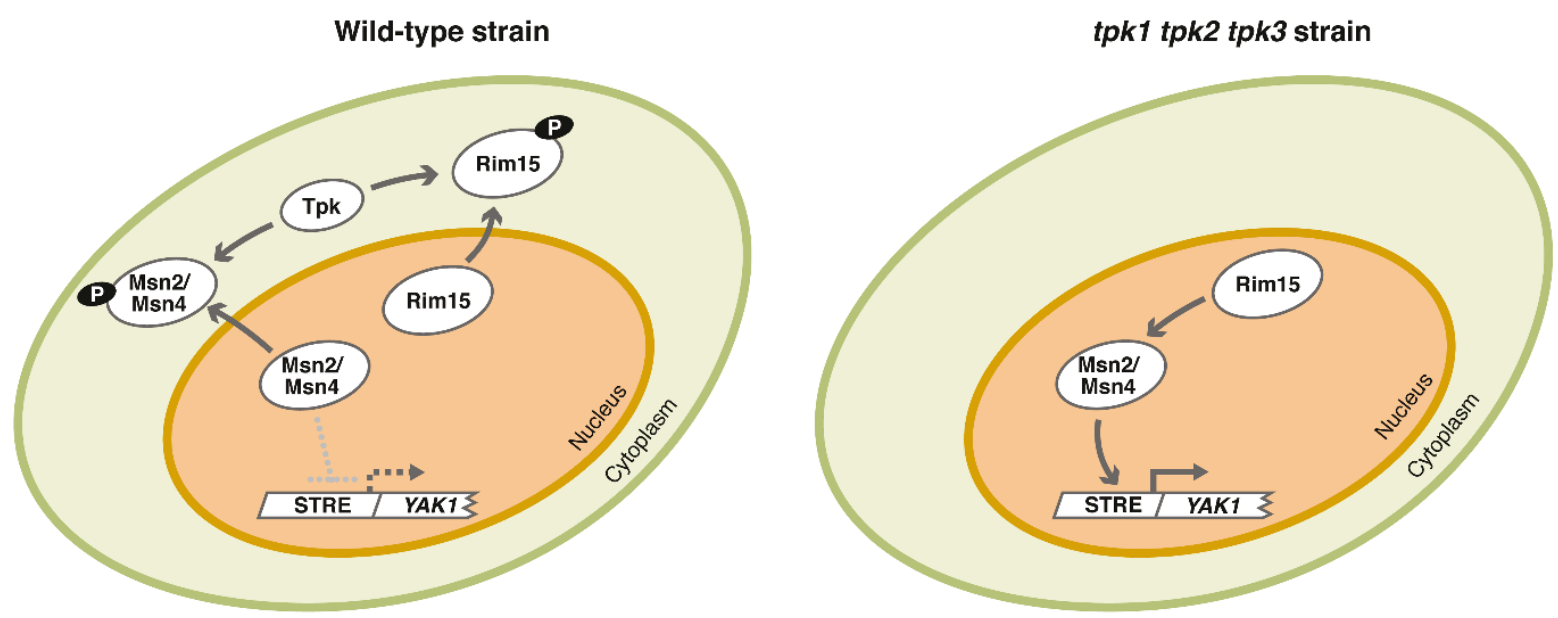

Figure 1 Relationships between Tpk1, 2, 3, Rim15, Msn2/4 and Yak1. Activated Tpks can phosphorylate Msn2/4 and Rim15 leading to their export to the cytoplasm, thus reducing the transcription rate of YAK1 and allowing growth. In the absence of Tpks, Rim15 and Msn2/4 are not phosphorylated and remain in the nucleus, in an active or potentially active form. Rim15 activates Msn2/4, Msn2/4 activate the transcription of YAK1 and Yak1 blocks cellular growth. 
genes showed at least a 1.8-fold increase of their transcript level and ca. 80 genes were induced by more than 4-fold. Among the genes induced by glucose, some were still strongly induced in at least one of the strains without functional TPK genes (induction factor of at least $70 \%$ of that measured in the reference strain), others were only partially induced, and still others did not show a transcriptional response to glucose (representative genes of each category are shown in Figure 2).
For some genes, such as $M I G 2$, the induction factor was different in the two strains lacking PKA (Figure 2). However, the observation that full induction takes place in one of the two strains was sufficient to indicate the existence of a glucose-signalling pathway independent of PKA. For genes such as HOR2, PKA may even attenuate the degree of glucose induction, which for this gene was higher in both strains lacking PKA (Figure 2).

Genes encoding proteins related with glucose metabolism (HXT3, PFK1, ENO1, ENO2, CDC19, PDC1, ADH1,

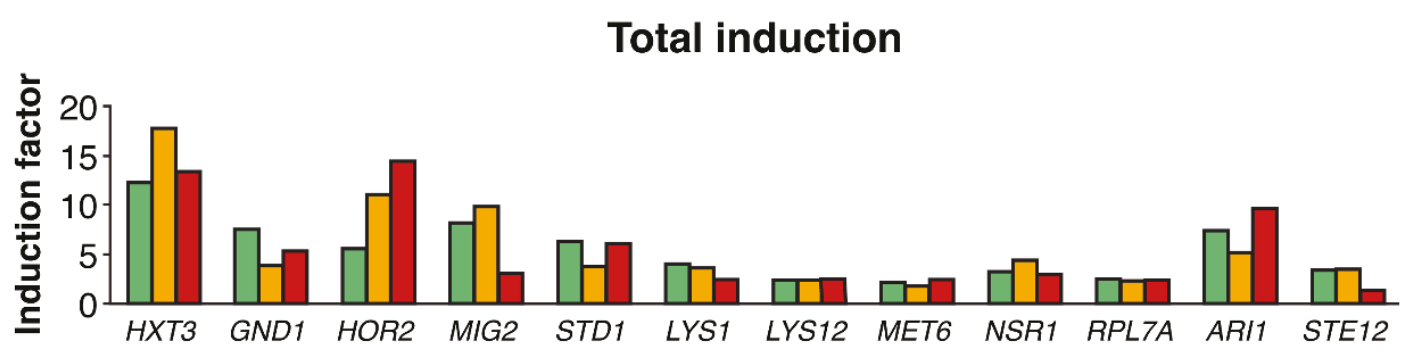

\section{Partial induction}

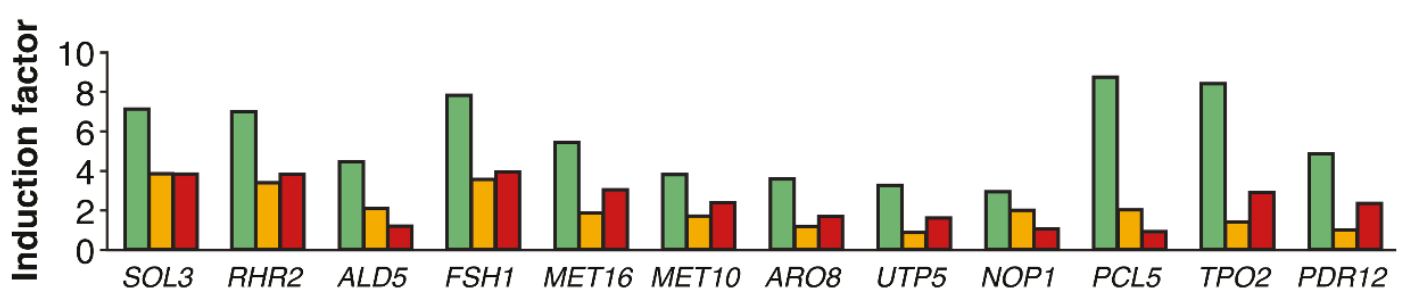

\section{Induction very low or absent}

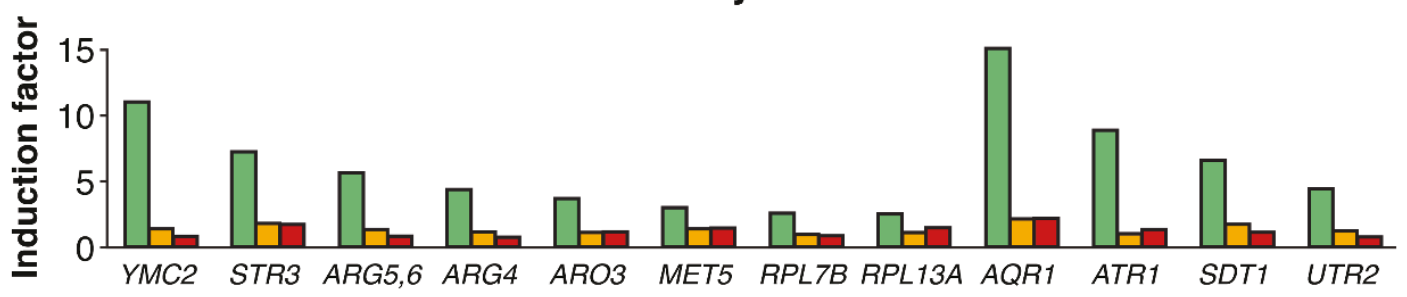

Figure 2 Consequences of the lack of PKA in the induction by glucose of some representative genes. Yeast strains TPK1 TPK2 TPK3, tpk1 tpk2 tpk3 msn2 msn4, and tpk1 tpk2 tpk3 yak1 were grown on ethanol and samples were taken before the addition of glucose to the medium and 30 min after, as described in Methods. mRNA levels were measured using Affymetrix microarrays. Biological duplicates were performed. Expression data from biological replicates generally differed from the average by less than $25 \%$. The induction factor is the quotient of the values measured in samples incubated in the presence of glucose and in those grown in the absence of glucose. Induction is considered "total" when the induction factor is no less than $70 \%$ of that measured in the reference strain in at least one of the strains lacking PKA. 
GND1, TKL1, HOR2, PMA1) mainly showed PKA-independent glucose induction, although in some cases the induction ratios decreased in the absence of PKA (ALD5, HXT1, PDC5, SOL3, RHR2) (Figure 2 and results not shown). Genes encoding diverse proteins that regulate carbon metabolism were also well induced in the absence of PKA (STD1, MIG2, MIG3) or partially induced (GCR1). For other categories of genes the results were not as clear-cut. For instance, for genes encoding proteins involved in amino acid biosynthesis we found that those in the lysine pathway (LYS1, LYS9, LYS12, LYS20) mostly showed PKA-independent glucose induction (with $L Y S 21$ as a notable exception), while the genes related with the arginine pathway (ARG1, ARG3, ARG4, ARG5,6, ARG7) were not induced by glucose in strains lacking PKA. Among the methionine biosynthesis genes, MET3, MET6 or MET32 did not require PKA for glucose induction, while in the absence of PKA induction was reduced for MET10, MET13 or MET16 and absent for MET5. Similarly, while most genes encoding ribosomal proteins and proteins involved in ribosome biogenesis ( $R P L 4 B, R P L 14 B, R P L 16 A, D B D 2, D B P 8$, NOG1, NOP16, NSR1 and many more) still responded to glucose in the absence of PKA, expression of four of them (RPL7B, RPL13A, RPS22B, NHP2) was not induced by glucose in the PKA-negative strains. Among multidrug membrane transporters, ATR1 was not induced by glucose in the absence of PKA, AQR1 was much less induced than in the wild-type strain and for PDR12 a 23 -fold decrease in the induction level was observed.

\section{Effects of PKA on induction by glucose during exponential growth}

For some yeast genes, induction by glucose is a transient phenomenon [5]. We, therefore, compared short-term induction by glucose and expression during exponential growth on glucose for a subset of genes, in strains with and without PKA (Table 1). To discard the possibility that differences could depend on repressing or inducing effects of ethanol, we tested cultures growing on glucose in the presence of ethanol. In the TPK1,2,3 reference strain we observed a strong induction of PDC1 by glucose, both in the short-term experiments and during exponential growth. For the other genes tested, induction ratios were more pronounced in the short-term experiments, an effect that was particularly marked for PDC5 (Table 1).

In the msn $2 m s n 4$ background, the absence of PKA had no effect on the induction of $P D C 1$, while for $P D C 5$ and $A L D 5$ only a weak effect in the short-term experiments was observed. For TMT1, YMC2 and ATR1 short-term induction was much reduced in the $t p k 1$ tpk2 tpk3 msn2 msn4 strain, but a 2-3-fold induction was still seen during exponential growth on glucose, similar to that observed in the wild-type strain. In the tpk1 tpk2 tpk3 yak1 strain, glucose induction was very much reduced for all six genes and absent in most cases (Table 1). This suggests a cooperative effect of the absence of PKA and the absence of Yak1, as all six genes were normally induced by glucose, although sometimes with a delay, in a yak1 strain (results not shown).

\section{Genes repressed by glucose}

Exposure to glucose causes transcriptional repression of a large number of $S$. cerevisiae genes. In the present study, short-term exposure to glucose of the TPK1 TPK2 TPK3 reference strain caused the repression of 500 genes by at least 4-fold and of 70 genes by at least 15 -fold. As we observed when studying glucose induction, some of these genes were also strongly repressed (repression factor of at least $70 \%$ of that measured in

Table 1 Short-term vs. Long-term effects of lack of PKA on induction of different genes by glucose

\begin{tabular}{|c|c|c|c|c|c|c|c|}
\hline \multirow[t]{3}{*}{ Strain } & \multirow[t]{3}{*}{ Growth conditions } & \multicolumn{6}{|c|}{ Gene } \\
\hline & & PDC1 & PDC5 & ALD5 & TMT1 & YMC2 & ATR1 \\
\hline & & \multicolumn{6}{|c|}{ Induction factor } \\
\hline \multirow[t]{2}{*}{ TPK1 TPK2 TPK3 } & 30 min with glucose & 12.8 & 235 & 5 & 9.7 & 11 & 13.8 \\
\hline & growth in medium with glucose & 27 & 1.3 & 3 & 2 & 4 & 2.3 \\
\hline \multirow{2}{*}{$\begin{array}{l}\text { tpk1 tpk2 tpk3 } \\
\text { msn2 msn4 }\end{array}$} & 30 min with glucose & 15 & 70 & 3.4 & 1.7 & 2.2 & 2.6 \\
\hline & growth in medium with glucose & 21 & 4.3 & 3.2 & 1.9 & 1.9 & 2.9 \\
\hline \multirow{2}{*}{$\begin{array}{l}\text { tpk1 tpk2 tpk3 } \\
\text { yak1 }\end{array}$} & 30 min with glucose & 1.2 & 1.8 & 0.9 & 1.1 & 0.8 & 1.4 \\
\hline & growth in medium with glucose & 4.4 & 2 & 0.6 & 0.7 & 0.7 & 0.9 \\
\hline
\end{tabular}

For short-term experiments yeasts were grown on ethanol as described in Methods and samples were taken before the addition of glucose to the medium and $30 \mathrm{~min}$ after. The yeasts were also grown on a medium containing $2 \%$ ethanol and $4 \%$ glucose and collected when over $50 \%$ of the glucose was still present in the medium. Biological duplicates were performed and mRNA levels were measured by RT-PCR as described. Expression data from biological replicates generally differed from the average by less than $25 \%$. The induction factor is the quotient of the values measured in samples grown in the presence of glucose and in the absence of glucose. 

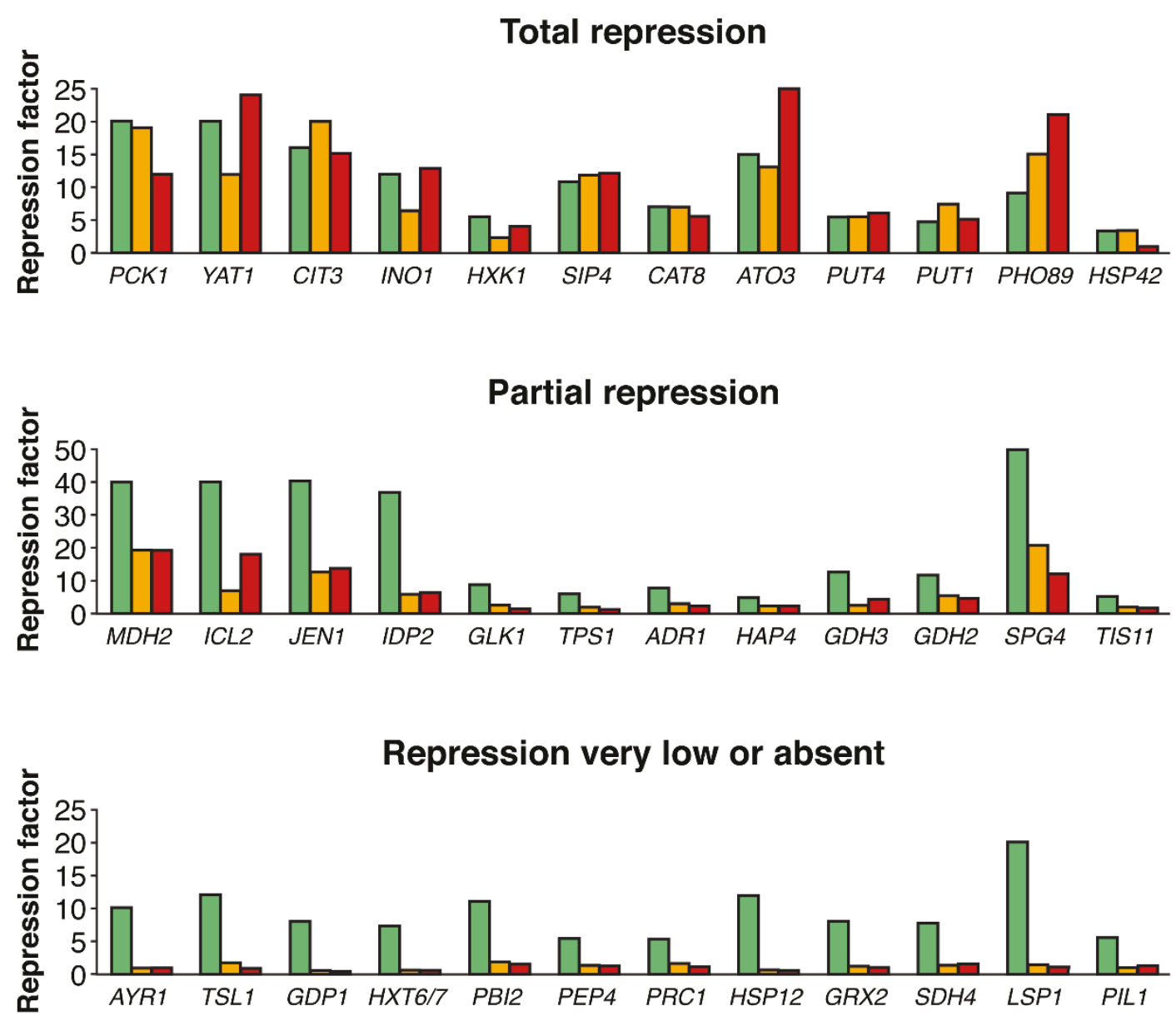

Figure 3 Consequences of the lack of PKA in the repression by glucose of some representative genes. Yeast strains TPK1 TPK2 TPK3, tpk1 tpk2 tpk3 msn2 msn4, and tpk1 tpk2 tpk3 yak1 were grown on ethanol and samples were taken before the addition of glucose to the medium and 30 min after, as described in Methods. mRNA levels were measured using Affymetrix microarrays. Biological duplicates were performed. Expression data from biological replicates generally differed from the average by less than $25 \%$. The repression factor is the quotient of the values measured in samples grown in the absence of glucose and in those incubated in the presence of glucose. Repression is considered "total" when the repression factor is no less than $70 \%$ of that measured in the reference strain in at least one of the strains lacking PKA.

the reference strain) in strains without a functional PKA, others were partially repressed, and still others not repressed at all in the PKA-negative strains. Representative expression profiles for each of these groups are shown in Figure 3.

Many of the genes required for growth on non-fermentable carbon sources were repressed by glucose in at least one of the two yeast strains without a functional PKA, either fully (ACS1, ADY2, CIT2, CIT3, DLD1, FBP1, ICL1, $M D H 2, M L S 1, P C K 1, S F C 1)$ or partially (ACH1, ACO1, ADH2, CIT1, COX7, CYB2, CYT1, FUM1, GUT2, IDH1, IDP2, JEN1, KGD1, KGD2, LSC1,
MDH1, MDH3) (Figure 3 and results not shown). Besides, genes encoding proteins that control carbon metabolism were repressed by glucose in the absence of PKA, with some showing full (CAT8, MAL33, REG2, SIP4, SNF3) and others partial (ADR1, HAP4, RGT1) repression.

Several genes encoding proteins with a variety of other functions were also strongly repressed by glucose in the absence of PKA. Their functions included glucose metabolism (HXK1, YIG1), lipid metabolism (CRC1, INO1, YAT1, YAT2), nitrogen metabolism (ATO3, PUT1, PUT4), phosphate metabolism (PHO89), and tolerance 
to high temperature (HSC82, HSP10, HSP42, HSP60, $S P G 4)$. Interestingly, many genes that were repressed by glucose in a PKA-negative background are known to be controlled by the transcription factor Cat8 (ACS1, ADY2, ATO3, FBP1, ICL1, JEN1, MDH2, MLS1, ODC1, PCK1, PUT4, SFC1, YAT2) [22] or to be direct targets of Ume6 (ACS1, ADY2, INO1, SIP4) [23] or Hap1 (CYB2, HXM1, MLS1, PUT1, PUT4) [24].

Of the genes that are no longer or hardly repressed by glucose, in the absence of PKA, many are related with respiration (COQ5, COX8, CYC3, CYT1, SDH1/2/3/4), ATP synthesis (ATP11, ATP14, ATP18, STF2), or stress responses (CTA1, DDR2, GRX2, HSP12, HYR1, PRX1, PST2, TPS1, TSA1, TSL1, TRX1). Others are involved in carbon metabolism (ALD4, ARA1, GPD1, HXT5/6/7, UGP1) or nitrogen metabolism, often in relation with proteolysis, (ALD3, AGX1, LAP4, PAI2, PBI2, PEP4, PRC1, RBD2).

\section{Effects of PKA on repression by glucose during exponential growth}

Most of the genes repressed by glucose in the shortterm experiments, particularly genes required for the utilization of alternative carbon sources, are known to be fully repressed during exponential growth on glucose [25]. We wondered whether genes involved in other cellular processes are subject to both short-term and longterm glucose repression. To address this question we selected some genes whose repression is strongly dependent on PKA. As shown in Table 2, we found that for TPS1 and TPS2, that encode the catalytic subunits of the trehalose-6-phosphate synthase/phosphatase complex, repression was transient and the same was observed for GRX2 and PEP4. In contrast, the HXT6/7 genes were still strongly repressed during growth, in a PKA-dependent manner.

\section{Impact of PKA on transcript profiles in gluconeogenic cultures}

During growth under gluconeogenic conditions, most yeast genes were expressed at similar levels in the TPK1 TPK2 TPK3 reference strain and in the two strains that lack a functional PKA. There was, however, a set of genes expressed at reduced levels in both mutant strains, and this set showed an overrepresentation of genes involved in stress responses, including thermal or oxidative stress (GPH1, GRE1, HSP26, HSP30, PMA2, SPG1, SPG4, YRO2, GAD1), osmotic shock (SIP18, STL1), and DNA damage (DDR48, YNL194c). Reduced expression of these genes was observed in both the msn 2 msn 4 and the yak1 genetic backgrounds, thus supporting the interpretation that PKA is required for their full expression under gluconeogenic conditions. We checked that most of these genes (except for DDR48, HSP30, SPG4, YNL194c) were also poorly expressed in a $t p k^{w}$ bcy 1 strain of $S$. cerevisiae grown under gluconeogenic conditions [5], an observation consistent with a dependence on PKA.

Although most genes showed a similar transcript level in the tpk1 tpk2 tpk3 msn2 msn4 and tpk1 tpk2 tpk3 yak1 strains, some notable exceptions were observed (Table 3). For the majority of genes expressed at lower levels in the tpk1 tpk2 tpk3 msn2 msn4 strain during growth on ethanol this is easily explained, as their expression has previously been reported to be activated by Msn2/4 [3]. The three remaining genes of this category (NCA3, STF2, YKL187c) are involved in some stress responses and have a mitochondrial function, but have no previously established relation with Msn2/4. Nevertheless, their behaviour is consistent with an as yet unidentified, and perhaps indirect, regulation by Msn2,4. For genes expressed at higher levels in the tpk1 tpk2 tpk3 msn $2 m s n 4$ strain or at higher or lower levels in

Table 2 Short-term vs. long-term effects of lack of PKA on repression of different genes by glucose

\begin{tabular}{|c|c|c|c|c|c|c|}
\hline \multirow[t]{3}{*}{ Strain } & \multirow[t]{3}{*}{ Growth conditions } & \multicolumn{5}{|c|}{ Gene } \\
\hline & & TPS1 & TPS2 & HXT6/7 & GRX2 & PEP4 \\
\hline & & \multicolumn{5}{|c|}{ Repression factor } \\
\hline \multirow[t]{2}{*}{ TPK1 TPK2 TPK3 } & 30 min with glucose & 7.7 & 7.1 & 30 & 9.5 & 15 \\
\hline & growth in medium with glucose & 1.3 & 1.6 & 68 & 2.9 & 1.8 \\
\hline \multirow{2}{*}{$\begin{array}{l}\text { tpk1 tpk2 tpk3 } \\
\text { msn2 msn4 }\end{array}$} & 30 min with glucose & 1.3 & 1.1 & 0.2 & 0.7 & 0.4 \\
\hline & growth in medium with glucose & 1.5 & 1.1 & 0.2 & 0.9 & 1 \\
\hline \multirow{2}{*}{$\begin{array}{l}\text { tpk1 tpk2 tpk3 } \\
\text { yak1 }\end{array}$} & 30 min with glucose & 1.1 & 1.3 & 1.4 & 1.4 & 1.2 \\
\hline & growth in medium with glucose & 0.3 & 0.4 & 0.3 & 0.5 & 0.6 \\
\hline
\end{tabular}


Table 3 Genes with different expression in strains tpk1 tpk2 tpk3 msn2 msn4 and tpk1 tpk2 tpk3 yak1 during growth on a gluconeogenic carbon source

\begin{tabular}{|c|c|c|c|}
\hline Gene & WT & tpk1 tpk2 tpk3 msn2 msn4 & tpk1 tpk2 tpk3 yak1 \\
\hline$H X K 1^{*}$ & 459 & 77 & 228 \\
\hline HSP104* & 327 & 121 & 229 \\
\hline $\mathrm{MSCl}^{*}$ & 629 & 58 & 296 \\
\hline $\mathrm{SOL4} 4^{*}$ & 148 & 28 & 107 \\
\hline YKL187C & 321 & 28 & 124 \\
\hline GLC3* & 390 & 96 & 425 \\
\hline GLK1* & 852 & 465 & 889 \\
\hline$G P X 1^{*}$ & 271 & 41 & 196 \\
\hline GSY2* & 184 & 45 & 245 \\
\hline HSP12* & 3790 & 807 & 2436 \\
\hline $\mathrm{HPF}^{*}$ & 650 & 137 & 611 \\
\hline NCA3 & 744 & 134 & 683 \\
\hline SOD ${ }^{*}$ & 1964 & 841 & 1558 \\
\hline STF2 & 838 & 183 & 1004 \\
\hline TPS1* & 569 & 245 & 612 \\
\hline TSL1* & 274 & 45 & 245 \\
\hline ATF2 & 31 & 33 & 173 \\
\hline PRM5 & 116 & 339 & 164 \\
\hline SSA2 & 997 & 1519 & 611 \\
\hline DAL3 & 198 & 403 & 58 \\
\hline FSHI & 220 & 271 & 68 \\
\hline
\end{tabular}

Yeast strains TPK1 TPK2 TPK3, tpk1 tpk2 tpk3 msn2 msn4, and tpk1 tpk2 tpk3 yak1, were grown on ethanol and samples were taken before the addition of glucose to the medium, as described in Methods. mRNA levels were measured using Affymetrix microarrays. Biological duplicates were performed. Expression data from biological replicates generally differed from the average by less than $20 \%$. Expression is shown in arbitrary units. (* Genes known to be activated by Msn2/4).

the tpk1 tpk2 tpk3 yak1 strain no immediate explanation is apparent.

\section{Discussion}

To bypass the lack of viability of $S$. cerevisiae strains lacking PKA, we have used strains lacking PKA and bearing suppressor mutations. Other, indirect, approaches have previously been used to study if PKA, or activation of PKA by CAMP, is required for the response of yeast to glucose. Slattery et al [26] used a cyr $1 \Delta$ strain, lacking adenylate kinase and therefore requiring $\mathrm{CAMP}$ for growth; the strain was grown in YPD + cAMP, starved for $24 \mathrm{~h}$ in spent YPD, resuspended in fresh YPD in the presence or absence of cAMP, and the changes produced in the transcriptome in these two conditions were compared. A different method was used by Zaman et al [27] to study the effects of blocking glucose signalling through PKA. In that work a $t p k 1^{a s} t p k 2^{a s} t p k 3^{a s}$ strain was built, with modified Tpks that had become sensitive to the inhibitor 1NM-PP1. Glucose was then added, together with the inhibitor, to a wild-type strain and to the mutant strain, both grown under gluconeogenic conditions, and the transcriptional responses of the two strains were compared. Our approach presents the advantage over the other two methods of allowing longterm experiments with growing cells (such as those shown in tables 1 and 2). It may also be noted that if phosphorylation of some protein by PKA, taking place in the absence of glucose, played a role in the transcriptional response to glucose, this would not be detected in the experiments performed by Zaman et al [27] where PKA activity was only blocked when glucose and the specific inhibitor were added to the cells. While avoiding the disadvantages associated with the use of inhibitors, the use of suppressor mutants can also present its own problems. In particular, the suppressor mutations may interfere with pathways related to the normal cellular responses to glucose or activate pathways that are not implicated in the response to glucose in wild-type backgrounds. In the present study, we have sought to minimize the risk of such complications by using strains with two different suppressor mutations.

Our results in the short-term experiments are generally consistent with the data presented by Zaman et al [27]. The main differences were found in some groups of genes induced by glucose. In our experiments the transcript levels of genes involved in glycolysis (PFK1, ENO1, ENO2, CDC19, ADH1) showed a small increase (40-80\%) both in the reference strain and in the strains lacking PKA. In contrast, Zaman et al [27] found that these genes were repressed by glucose in the reference strain (2 to 6-fold). In their experiments blocking PKA eliminated this repression, allowing a slight induction $(20-80 \%)$ in some cases. Induction by glucose of genes related with ribosome biogenesis in the reference strain was similar in the two sets of experiments; however, in our conditions most of these genes were induced in the absence of PKA, while in the work of Zaman et al. [27] inhibition of PKA impaired their induction. Since $S$. cerevisiae strains with the W303 genetic background were used in both studies, it seems likely that the disparities observed are related with the different media used for growth. Zaman et al. [27] used glycerol and ammonium as carbon and nitrogen source while our cultures contained ethanol and glutamate.

As shown in Tables 1 and 2, for a number of genes the transcriptional response to glucose is transient. This may be explained by the fact that the large increase in intracellular cAMP, sometimes referred as the cAMP "spike", observed upon exposure of the yeast cells to glucose is itself transient [28]. It could be expected that for genes that respond to glucose independently of PKA the response would be long- 
lived and, in fact, induction of PDC1 is maintained or even increased during exponential growth (Table 1). For ALD5, TMT1, YMC2 and ATR1 two signalling pathways appear to be operative, one of them independent of PKA and acting both shortly after glucose addition and during growth, the other one depending on PKA and with transient effects. The behaviour of PDC5 could be explained by the operation of a PKAindependent system whose effects are transient. We have also observed that for some genes, that show no response to the sugar in the absence of PKA, repression by glucose in the wild-type strain is transient (Table 2).

Our observations on the involvement of PKA in induction and repression by glucose, together with the results of Wang et al [5], allow the classification of genes responding to glucose in four categories (Table 4).

\section{Genes in class 1 are controlled exclusively by signalling pathway(s) independent of PKA}

These genes can be induced (or repressed) in the absence of PKA and are not induced (or repressed) upon activation of PKA in the absence of glucose. In this category, induced genes are mostly related with glucose metabolism, while repressed genes are involved in utilization of alternative carbon sources.

\section{Genes in class 2 are controlled only by signalling pathway} (s) dependent on PKA

These genes can be induced/repressed by activation of PKA and do not respond to glucose in the absence of PKA. Many of the induced genes in this category encode proteins involved in amino acid biosynthesis, while the repressed genes form a heterogeneous group.

Genes in class 3 are controlled by redundant PKA-

\section{dependent and PKA-independent signalling pathways}

These genes can be fully induced/repressed by activation of PKA, but also by glucose in the absence of PKA. Among the induced genes in this category, those encoding glucose transporters and ribosomal proteins are prevalent, while many of the repressed genes are involved in the utilization of non-fermentable carbon sources.

Genes in class 4 are controlled by cooperative signalling pathways, of which at least one is PKA-dependent

These genes encode proteins of different types. Some of them do not respond to the activation of PKA nor to the addition of glucose in the absence of PKA. For others, a partial induction/repression may be observed in one of these conditions or in both.

What could be the advantages to the cell of employing these different modes of regulation? While control through a single signal may be adequate in many cases, regulation of genes by redundant signalling pathways could confer a greater robustness to their response to glucose. On the other hand, regulation by several cooperative pathways that, individually, cannot give a full transcriptional response, could provide a greater flexibility, as discussed by Chen and Thorner [29] for the case of yeast responses to nutrient limitation.

The next important goal will be to establish the PKAdependent and PKA-independent pathways operating in each case, since information on this topic is still very incomplete. A notable exception is the PKA-independent pathway controlling the glucose-induced $H X T$ genes that encode glucose transporters. The plasma membrane proteins Snf3/Rgt2 act as sensors for external glucose and, when bound to the sugar, initiate a chain of reactions that results in the dissociation of the repressor Rgt1 from the promoters of the corresponding $H X T$ genes [30,31]. For other types of genes induced by glucose the situation is less clear. The protein kinase Snf1 may play a role in the case of genes encoding enzymes involved in amino acid biosynthesis, since Snf1 represses the translation of the transcriptional activator Gcn4 [32]. As glucose inactivates Snf1 [33], this would result in the induction of the genes activated by Gcn 4 .

Table 4 Involvement of PKA in the transcriptional regulation of different classes of genes controlled by glucose

\begin{tabular}{|c|c|c|c|c|c|}
\hline \multirow[t]{2}{*}{ Class } & \multirow{2}{*}{$\begin{array}{c}\text { Transcriptional } \\
\text { effect }\end{array}$} & \multicolumn{2}{|c|}{ Glucose added to strain } & \multirow{2}{*}{$\begin{array}{c}\text { No glucose added } \\
\text { PKA activated }\end{array}$} & \multirow{2}{*}{$\begin{array}{l}\text { Representative } \\
\text { genes }\end{array}$} \\
\hline & & PKA $^{+}$ & PKA $^{-}$ & & \\
\hline \multirow[t]{2}{*}{1} & Induction & + & + & - & PFK1, ENO2 \\
\hline & Repression & + & + & - & $C R C 1, P C K 1, M L S 1$ \\
\hline \multirow[t]{2}{*}{2} & Induction & + & - & + & ARG3, THR4 \\
\hline & Repression & + & - & + & GRX2, PEP4 \\
\hline \multirow[t]{2}{*}{3} & Induction & + & + & + & HXT3, RPL7A, HOR2 \\
\hline & Repression & + & + & + & ACS1, ADY2, CIT3 \\
\hline \multirow[t]{2}{*}{4} & Induction & + & - & - & SDT1, STR3 \\
\hline & Repression & + & - & - & HXT6, MDH1 \\
\hline
\end{tabular}

The presence or absence of transcriptional response after addition of glucose to yeast strains with or without PKA activity is indicated by + or -. The column labelled "No glucose added" shows the transcriptional response when PKA is activated through an increase in cAMP that does not involve the presence of glucose [5]. 
Another important group of glucose-induced genes encodes proteins involved in the biogenesis of ribosomes and is subject to a very complex combinatorial control. Binding, through Rap1, of the transcription factor Fhl1 together with the coactivator Ifh1 to the corresponding promoters activates the transcription of these genes, while other factors, Cfr1, Dot6 and Tod6, Stb3, contribute to repress their transcription. Glucose modifies the intracellular localization of these factors: it promotes a cytoplasmic location for Cfr1 [34], Dot6 and Tod6 [35], and Stb3 [36] and the nuclear localization of Fhl1 and Ifh1 [37]. While PKA can phosphorylate Dot6 and Tod6 and this is likely to direct them to the cytoplasm, export from the nucleus of Stb3 depends on Tor activity but not on PKA. Glucose also promotes the phosphorylation by PKA of the global transcriptional regulator $S f p 1$, responsible for the nuclear relocalization of Fhl1 and Ifh1. However, phosphorylation by PKA of Sfp1, in the absence of glucose, is not sufficient to direct Sfp1 to the nucleus [37]. This indicates that an alternative signalling pathway is involved in the process; it may be Tordependent, since there is already evidence for activation by glucose of the Tor signalling pathway $[36,38,39]$. However, the molecular mechanism responsible for this effect of glucose on Tor has not been yet established. With respect to genes encoding glycolytic enzymes, they depend on the transcription factors Gcr1 and Gcr2 $[40,41]$ but there is no information on the regulation of these factors by glucose.

Most of the genes repressed by glucose depend for their transcription on the activity of the protein kinase Snf1 and/or on the transcription factors Hap1, Hap4, Cat 8 and Adr1 [27]. There is no evidence that Hap1 itself is regulated by glucose; repression by glucose of genes activated by Hap1 depends on its control of other transcription factors such as Hap4 or Adr1 [42]. Transcription of HAP4 and ADR1 is repressed by glucose and we have found that the degree of repression decreases in the absence of PKA, but the intimate mechanism of the repression of transcription is unknown. Snf1 is not required for the induction of ADR1 [43] or of HAP4 [27], that takes place in the absence of glucose, but a Snf1-dependent pathway is responsible for the activation of the transcription factor Adr1 in these conditions [44]. Snf1 is also necessary both for the transcription of CAT8 and for the transcriptional activation of Cat8 [45]. We have observed that glucose represses SNF1 transcription about 2-fold, in addition to its well-known role in decreasing $\mathrm{Snf} 1$ activity [33]. Although the mechanism for this decrease is not established, it has been proposed that the $\beta$ subunit of the Snf1 complex is important to maintain Snf1 in an inactive state [46]. Specifically, the glycogen-binding domain of the $\beta$ subunit Gal83 would mediate the recruitment of the Reg1-Glc7 complex, responsible for the dephosphorylation and subsequent inactivation of Snf1.

It should be stressed that, even in cases where PKA has been shown to play an important role in the control of gene transcription by glucose, the target for PKA has not been generally identified. A possible approach for clarifying the role of PKA could be to check whether selected proteins identified as potential PKA substrates participate in the glucose response. Surprisingly, among the many proteins interacting with Tpk1, Tpk2 or Tpk3 and characterized as possible substrates $[47,48]$ few good candidates appeared. The proteins Msn2/Msn4 are an interesting exception. For the genes under control of Msn2/Msn4, PKA operates directly through the phosphorylation of the transcription factors, allowing their export to the cytoplasm, and thus precluding transcription. As shown in table 5, expression of the relevant genes is already low in the absence of glucose in the strain lacking Msn2/Msn4 and the effect of glucose is modest. In the tpk1 tpk2 tpk3 yak1 strain expression is similar to that of the wild-type strain under gluconeogenic conditions but it is much less sensitive to the addition of glucose, as the transcriptional activators remain in the nucleus. New strategies would have to be devised to identify additional protein substrates of PKA that may play a role in the response to glucose.

\section{Conclusions}

This study shows that, although PKA is frequently involved in the control of transcription by glucose, there are many instances in which PKA activity is dispensable. We have also observed that for genes regulated exclusively through PKA the effect of glucose on transcription may be transient. It may be concluded that the role of cAMP/PKA in transcription, which was first identified a couple of decades ago, still represents many challenges and that one of the goals of systems biology, the

Table 5 Genes activated by Msn2/Msn4 and depending on PKA for glucose repression

\begin{tabular}{|c|c|c|c|c|c|c|}
\hline \multirow[t]{3}{*}{ Gene } & \multicolumn{6}{|c|}{ Relevant genotype } \\
\hline & \multicolumn{2}{|c|}{$\begin{array}{c}\text { TPK1 TPK2 } \\
\text { TPK3 }\end{array}$} & \multicolumn{2}{|c|}{$\begin{array}{c}\text { tpk1 tpk2 tpk3 msn2 } \\
\text { msn4 }\end{array}$} & \multicolumn{2}{|c|}{$\begin{array}{c}\text { tpk1 tpk2 tpk3 } \\
\text { yak1 }\end{array}$} \\
\hline & $\mathrm{Et}$ & + Glu & Et & + Glu & Et & + Glu \\
\hline GPX1 & 100 & 6 & 15 & 8 & 73 & 45 \\
\hline GSY2 & 100 & 13 & 23 & 15 & 80 & 62 \\
\hline HPF1 & 100 & 41 & 21 & 42 & 94 & 108 \\
\hline MSC1 & 100 & 4 & 9 & 6 & 45 & 39 \\
\hline TSL 1 & 100 & 8 & 16 & 9 & 90 & 92 \\
\hline
\end{tabular}

A wild-type strain and two strains lacking PKA were grown on ethanol, samples taken before (Et) and 30 min after the addition of glucose (+Glu), and mRNA levels measured (see Methods for details). Expression is given as percent of the value in the wild-type strain growing on ethanol. 
Table 6 S. cerevisiae strains used in this work

\begin{tabular}{|c|c|c|}
\hline Strain & Genotype & Reference \\
\hline $\begin{array}{l}\text { W303-1A } \\
\text { W303-1B }\end{array}$ & $\begin{array}{l}\text { MATa ade2-1 his3-11,15 leu2-3, } 112 \text { trp1-1 ura3-1 can1-100 TPK1 TPK2 TPK3 } \\
\text { MATa ade2-1 his3-11,15 leu2-3, } 112 \text { trp1-1 ura3-1 can1-100 TPK1 TPK2 TPK3 }\end{array}$ & $\begin{array}{l}49] \\
{[49]}\end{array}$ \\
\hline W yak1 & MAT $\alpha$ ade2-1 his3-11,15 leu2-3, 112 trp1-1 ura3-1 can1-100 yak1::KanMX TPK1 TPK2 TPK3 & [14] \\
\hline $\begin{array}{l}\text { W tpk1 tpk2 tpk3 msn2 } \\
\text { msn4 }\end{array}$ & $\begin{array}{c}\text { MATa ade2-1 his3-11,15 leu2-3, } 112 \text { trp 1-1 ura 3-1 can1-100 tpk1::URA3 tpk2::HIS3 tpk3::TRP1 msn2::HIS3 msn4:: } \\
\text { TRP1 }\end{array}$ & [50] \\
\hline $\begin{array}{l}\text { W tpk1 tpk2 tpk3 } \\
\text { yak1 }\end{array}$ & MAT $\alpha$ ade2-1 his3-11,15 leu2-3, 112 trp1-1 ura3-1 can1-100 tpk1::URA3 tpk2::HIS3 tpk3::TRP1 yak1::LEU2 & [51] \\
\hline CJM567 & MATa can1-100 TPK1 TPK2 TPK3 & This work \\
\hline CJM573 & MAT $\alpha$ can1-100 yak1::KanMX TPK1 TPK2 TPK3 & This work \\
\hline CJM569 & MATa his3-11,15 ura3-1, 112 trp1-1 can1-100 tpk1::URA3 tpk2::HIS3 tpk3::TRP1 msn2::HIS3 msn4::TRP1 & This work \\
\hline CJM571 & MATa his3-11,15 leu2-3, 112 ura3-1 trp1-1 can1-100 tpk1::URA3 tpk2::HIS3 tpk3::TRP1 yak1::LEU2 & This work \\
\hline
\end{tabular}

construction of predictive models of signal transduction pathways, requires additional detailed studies on the mechanism(s) by which PKA is involved in the transcriptional regulation of a substantial part of the yeast genome and of the context dependency of these mechanisms.

\section{Methods}

\section{Yeast strains and growth conditions}

The yeast strains used are listed in Table 6. All of them are derived from strains W303-1A and W303-1B [49]. The prototrophic strains CJM 567, CJM 573, CJM 569 and CJM 571 were constructed by substituting in the original strains, W303-1A, W yak1 [14], W303 tpk123 msn2 msn4 [50] and W303 tpk123 yak1 [51], through successive transformations, the mutated genes (ade2, his3, leu2, trp1, ura3) by their wild-type alleles, as needed, using selective media. The mutant strains, CJM 573, CJM 569 and CJM 571 were checked by diagnostic PCR.

Yeasts were grown at $30^{\circ} \mathrm{C}$ in minimal media (YNB with $0.1 \%$ sodium glutamate as nitrogen source) with $2 \%$ ethanol or $4 \%$ glucose $+2 \%$ ethanol as carbon sources and collected at the early exponential phase of growth. Samples were also taken after adding glucose (end concentration $4 \%$ ) to cultures in $2 \%$ ethanol and incubating them at $30^{\circ} \mathrm{C}$ for $30 \mathrm{~min}$.

\section{Sampling and RNA isolation}

Yeast cells were collected by rapid filtration [52] and stored at $-70^{\circ} \mathrm{C}$ until use. The RNA to be used for the microarrays was extracted using the hot-phenol method [53]. For the RT-qPCR measurements RNA was extracted with glass beads and the TRIzol LS reagent (Invitrogen, Carlsbad, CA, USA) as described [52] and quantified using the Thermo Scientific NanoDrop Spectrophotometer.

\section{Probe preparation and hybridization to arrays}

cDNA synthesis, cRNA synthesis and labelling, as well as array hybridization were performed as described [54].

\section{Data acquisition and primary analysis}

Acquisition and quantification of the Affymetrix yeast S98 array images as well as primary data analysis were performed using the Affymetrix GeneChip Operating Software (GCOS) 2.1. All arrays were globally scaled to a target value of 150 using the average signal from all

Table 7 Probes used for the RT-qPCR measurements

\begin{tabular}{cllc}
\hline Gene & Direct oligonucleotide & Reverse oligonucleotide & Product(bp) \\
\hline PDC1 & TAGAACTCCAGCTAACGCTGCTGT (1053-1076) & TGGGAAAGTGGTTGGTTGATACC (1180-1203) & 151 \\
PDC5 & GCCAAGTCAACTGTAACACCGTCT (44-68) & CAGCATAGGCAGCGTCAATCGT (149-173) & 130 \\
HXT6 & GGGCTGTTGGTCTCATGTTCTTC (1497-1521) & CATTCTTCAGCGTCGTAGTGGC (1642-1665) & 169 \\
ALD5 & AGCCAACAGTGTTGCTGATGTCAA (1205-1229) & GCGGCTAACCCATATTGAGAATCAT (1322-1346) & 142 \\
TPS1 & TCTCGTCCACCCGTGATGGTATGAA (1301-1325) & ATGGCATCAGAAAGATCATCGGTG (1160-1164) & 166 \\
TPS2 & GGGCAACTACGGATTCTATCCTGTC (2430-2454) & TCTGGAATCCAGGTCGACCGTACCA(2568-2592) & 163 \\
ATR1 & TAATAGTAGAGCCTTCCAAGGGC (494-517) & CAACCTAAAGTTCGTCCAATAGGG (601-647) & 156 \\
GRX2 & CTACTCCAAAAATGGTATCCCAGG (92-115) & GGAACGTCAATTCTTGAAGAGG (210-233) & 144 \\
PEP4 & GATAAGGTGGTCCCTCCATTTAC (622-645) & AACAGGTAACCAAGTGATATCGCC (769-792) & 173 \\
TMT1 & TGATTGAAGTCCTACGGGAAGC (494-517) & CTTGTCTCTCACATCTTCTGCAC (629-652) & 161 \\
YMC2 & GTTGACAGTATACCCATTGGACGTT (762-786) & CCAAACCCTTGAAAAAGGCTCTGA (881-905) & 146 \\
\hline
\end{tabular}

The oligonucleotides were checked for specificity using the BLAST feature of SGD. 
gene features. The Significance Analysis of Microarrays (SAM version 3.0) add-in to Microsoft Excel was used for comparisons of duplicate array experiments [55] SAM assesses the difference between two mean values when taking into account the standard errors of those means. The significance of that difference is estimated by comparing it against the probability of its occurrence by chance alone. The model of chance occurrence is generated by permutation of the input data, rather than a predetermined model (e.g. a normal distribution), as is used by the $t$-test.

The transcript data can be downloaded from Genome Expression Omnibus (http://www.ncbi.nlm.nih.gov/geo) under the series accession number GSE27541.

\section{RT-qPCR measurements}

The RNA samples were treated with DNase I (Ambion) and the quality of the purified RNA checked using the Agilent 2100 Bioanalyzer. The oligonucleotides used for RT-qPCR are shown in table 7. The measurements were performed in triplicate using SYBR green I. Normalization was carried out with the $18 \mathrm{~S}$ rRNA.

\section{List of abbreviations}

PCR: polymerase chain reaction; PKA: protein kinase $A ;$ RT-qPCR: quantitative real-time polymerase chain reaction; YNB: Yeast Nitrogen Base.

\section{Acknowledgements}

We thank Carlos Gancedo for a thorough revision of the manuscript and Cecile Brocard (University of Vienna) for kindly providing yeast strains. This work was supported by Grant BFU2007-66063-C02-01/BMC from the Spanish Ministry of Science and Innovation (MICINN). DL was a pre-doctoral fellow from the Conacyt (Mexico). The Kluyver Centre for Genomics of Industrial Fermentation is sponsored by the Netherlands Genomics Initiative.

\section{Author details}

'Department of Metabolism and Cell Signalling, Instituto de Investigaciones Biomédicas Alberto Sols, CSIC-UAM, Arturo Duperier 4, 28029 Madrid, Spain. ${ }^{2}$ Department of Biotechnology, Delft University of Technology and Kluyver Centre for Genomics and Industrial Fermentation, Julianalaan 67, 2628 BC Delft, The Netherlands.

\section{Authors' contributions}

DL performed the cultures, collected the yeast samples and extracted RNA. MA performed the microarray experiments. JMD analysed the microarray data. JTP co-designed the experiments and contributed to the writing. JMG conceived the study, designed experiments, constructed yeast strains, analysed data and wrote the paper. All authors discussed the results and read and approved the final manuscript.

Received: 15 April 2011 Accepted: 9 August 2011

Published: 9 August 2011

\section{References}

1. van der Plaat JB: Cyclic $3^{\prime}, 5^{\prime}$-adenosine monophosphate stimulates trehalose degradation in baker's yeast. Biochem Biophys Res Commun 1974, 56:580-587.

2. Mazón MJ, Gancedo JM, Gancedo C: Phosphorylation and inactivation of yeast fructose-bisphosphatase in vivo by glucose and by proton ionophores. A possible role for cAMP. Eur J Biochem 1982, 127:605-608.

3. Boy-Marcotte E, Perrot M, Bussereau F, Boucherie H, Jacquet M: Msn2 and Msn4 control a large number of genes induced at the diauxic transition which are repressed by cyclic AMP in Saccharomyces cerevisiae. $J$ Bacteriol 1998, 180:1044-1052.

4. Zaragoza O, Lindley C, Gancedo JM: Cyclic AMP can decrease expression of genes subject to catabolite repression in Saccharomyces cerevisiae. J Bacteriol 1999, 181:2640-2642.

5. Wang Y, Pierce M, Schneper L, Guldal CG, Zhang X, Tavazoie S, Broach JR: Ras and Gpa2 mediate one branch of a redundant glucose signaling pathway in yeast. PLOS Biol 2004, 2:610-622.

6. Toda T, Uno I, Ishikawa T, Powers S, Kataoka T, Broek D, Cameron S, Broach J, Matsumoto K, Wigler M: In yeast, RAS proteins are controlling elements of adenylate cyclase. Cell 1985, 40:27-36.

7. Kübler E, Mösch H-U, Rupp S, Lisanti MP: Gpa2p, a G-protein a-subunit, regulates growth and pseudohyphal development in Saccharomyces cerevisiae via a cAMP-dependent mechanism. J Biol Chem 1997, 272:20321-20323.

8. Colombo S, Ronchetti D, Thevelein JM, Winderickx J, Martegani E: Activation state of the Ras2 protein and glucose-induced signaling in Saccharomyces cerevisiae. J Biol Chem 2004, 279:46715-46722.

9. Paiardi C, Belotti F, Colombo S, Tisi R, Martegani E: The large N-terminal domain of $\mathrm{Cdc} 25$ protein of the yeast Saccharomyces cerevisiae is required for glucose-induced Ras2 activation. FEMS Yeast Res 2007, 7:1270-1275.

10. Yun CW, Tamaki H, Nakayama R, Yamamoto K, Kumagai H: G-protein coupled receptor from yeast Saccharomyces cerevisiae. Biochem Biophys Res Commun 1997, 240:287-292.

11. Xue $Y$, Battle $M$, Hirsch JP: GPR1 encodes a putative $G$ protein-coupled receptor that associates with the Gpa2p $G_{\alpha}$ ubunit and functions in a Ras-independent pathway. EMBO J 1998, 17:1996-2007.

12. Rolland F, De Winde J, Lemaire K, Boles E, Thevelein J, WinderickX J: Glucose-induced cAMP signalling in yeast requires both a G-protein coupled receptor system for extracellular glucose detection and a separable hexose kinase-dependent sensing process. Mol Microbiol 2000, 38:348-358.

13. Toda T, Cameron S, Sass P, Zoller M, Wigler M: Three different genes in S. cerevisiae encode the catalytic subunits of the CAMP-dependent protein kinase. Cell 1987, 50:277-287.

14. Garrett S, Broach J: Loss of Ras activity in Saccharomyces cerevisiae is suppressed by disruption of a new kinase gene, YAK1, whose product may act downstream of the CAMP-dependent protein kinase. Genes Dev 1989, 3:1336-1348.

15. Reinders A, Burckert N, Boller T, Wiemken A, De Virgilio C: Saccharomyces cerevisiae CAMP-dependent protein kinase controls entry into stationary phase through the Rim15p protein kinase. Genes Dev 1998, 12:2943-2955.

16. Smith A, Ward M, Garrett S: Yeast PKA represses Msn2p/Msn4pdependent gene expression to regulate growth, stress response and glycogen accumulation. Embo J 1998, 17:3556-3564.

17. Hartley A, Ward M, Garrett S: The Yak1 protein kinase of Saccharomyces cerevisiae moderates thermotolerance and inhibits growth by an Sch9 protein kinase-dependent mechanism. Genetics 1994, 136:465-474.

18. Moriya H, Shimizu-Yoshida Y, Omori A, Iwashita S, Katoh M, Sakai A: Yak1p, a DYRK family kinase, translocates to the nucleus and phosphorylates yeast Pop2p in response to a glucose signal. Genes Dev 2001, 15:1217-1228.

19. Görner W, Durchschlag E, Martínez-Pastor M, Estruch F, Ammerer G, Hamilton B, Ruis $\mathrm{H}$, Schüller $\mathrm{C}$ : Nuclear localization of the $\mathrm{C}_{2} \mathrm{H}_{2}$ zinc finger protein Msn2p is regulated by stress and protein kinase $A$ activity. Genes Dev 1998, 12:586-597.

20. Cameroni E, Hulo N, Roosen J, Winderickx J, De Virgilio C: The novel yeast PAS kinase Rim15 orchestrates G0-associated antioxidant defense mechanisms. Cell Cycle 2004, 3:462-468.

21. Lee P, Cho BR, Joo HS, Hahn JS: Yeast Yak1 kinase, a bridge between PKA and stress-responsive transcription factors, $\mathrm{Hsf1}$ and Msn2/Msn4. Mol Microbiol 2008, 70:882-895.

22. Tachibana C, Yoo JY, Tagne JB, Kacherovsky N, Lee TI, Young ET: Combined global localization analysis and transcriptome data identify genes that are directly coregulated by Adr1 and Cat8. Mol Cell Biol 2005, 25:2138-2146.

23. Williams RM, Primig M, Washburn BK, Winzeler EA, Bellis M, Sarrauste de Menthiere C, Davis RW, Esposito RE: The Ume6 regulon coordinates metabolic and meiotic gene expression in yeast. Proc Natl Acad Sci USA 2002, 99:13431-13436. 
24. Ter Linde JJ, Steensma HY: A microarray-assisted screen for potential Hap1 and Rox1 target genes in Saccharomyces cerevisiae. Yeast 2002, 19:825-840.

25. DeRisi JL, lyer VR, Brown PO: Exploring the metabolic and genetic control of gene expression on a genomic scale. Science 1997, 278:680-686.

26. Slattery MG, Liko D, Heideman W: Protein kinase A, TOR, and glucose transport control the response to nutrient repletion in Saccharomyces cerevisiae. Eukaryot Cell 2008, 7:358-367.

27. Zaman S, Lippman Sl, Schneper L, Slonim N, Broach JR: Glucose regulates transcription in yeast through a network of signaling pathways. Mol Syst Biol 2009, 5:245-259.

28. Eraso P, Gancedo JM: Use of glucose analogues to study the mechanism of glucose-mediated CAMP increase in yeast. FEBS Lett 1985, 191:51-54.

29. Chen RE, Thorner J: Systematic epistasis analysis of the contributions of protein kinase A- and mitogen-activated protein kinase-dependent signaling to nutrient limitation-evoked responses in the yeast Saccharomyces cerevisiae. Genetics 2010, 185:855-870.

30. Özcan S, Jonhston M: Function and regulation of yeast hexose transporters. Microbiol Mol Biol Rev 1999, 63:554-569.

31. Gancedo JM: The early steps of glucose signalling in yeast. FEMS Microbiol Rev 2008, 32:673-704.

32. Shirra MK, McCartney RR, Zhang C, Shokat KM, Schmidt MC, Arndt KM: A chemical genomics study identifies Snf1 as a repressor of GCN4 translation. J Biol Chem 2008, 283:35889-35898.

33. Carlson M: Glucose repression in yeast. Curr Opinion Microbiol 1999, 2:202-207.

34. Martin DE, Soulard A, Hall MN: TOR regulates ribosomal protein gene expression via PKA and the Forkhead transcription factor FHL1. Cell 2004, 119:969-979.

35. Lippman SI, Broach JR: Protein kinase A and TORC1 activate genes for ribosomal biogenesis by inactivating repressors encoded by Dot6 and its homolog Tod6. Proc Natl Acad Sci USA 2009, 106:19928-19933.

36. Liko D, Conway MK, Grunwald DS, Heideman W: Stb3 plays a role in the glucose-induced transition from quiescence to growth in Saccharomyces cerevisiae. Genetics 2010, 185:797-810.

37. Jorgensen P, Rupes I, Sharom JR, Schneper L, Broach JR, Tyers M: A dynamic transcriptional network communicates growth potential to ribosome synthesis and critical cell size. Genes Dev 2004, 18:2491-2505.

38. Peter GJ, During L, Ahmed A: Carbon catabolite repression regulates amino acid permeases in Saccharomyces cerevisiae via the TOR signaling pathway. J Biol Chem 2006, 281:5546-5552.

39. Urban J, Soulard A, Huber A, Lippman S, Mukhopadhyay D, Deloche O, Wanke $V$, Anrather D, Ammerer G, Riezman H, et al: Sch9 is a major target of TORC1 in Saccharomyces cerevisiae. Mol Cell 2007, 26:663-674.

40. Uemura H, Fraenkel DG: gcr2, a new mutation affecting glycolytic gene expression in Saccharomyces cerevisiae. Mol Cell Biol 1990, 10:6389-6396.

41. Chambers A, Packham E, Graham I: Control of glycolytic gene expression in the budding yeast (Saccharomyces cerevisiae). Curr Genet 1995, 29:1-9.

42. Ramil E, Agrimonti C, Shechter E, Gervais M, Guiard B: Regulation of the CYB2 gene expression: transcriptional co-ordination by the Hap1p, Hap2/3/4/5p and Adr1p transcription factors. Mol Microbiol 2000, 37:1116-1132.

43. Dombek KM, Young ET: Cyclic AMP-dependent protein kinase inhibits $A D H 2$ expression in part by decreasing expression of the transcription factor gene ADR1. Mol Cell Biol 1997, 17:1450-1458.

44. Ratnakumar S, Kacherovsky N, Arms E, Young ET: Snf1 controls the activity of Adr1 through dephosphorylation of Ser230. Genetics 2009, 182:735-745.

45. Rahner A, Schöler A, Martens E, Gollwitzer B, Schüller H-J: Dual influence of the yeast Cat1p (Snf1p) protein kinase on carbon source-dependent transcriptional activation of gluconeogenic genes by the regulatory gene CAT8. Nucleic Acids Res 1996, 24:2331-2337.

46. Mangat S, Chandrashekarappa D, McCartney RR, Elbing K, Schmidt MC: Differential roles of the glycogen-binding domains of $\beta$ subunits in regulation of the Snf1 kinase complex. Eukaryot Cell 2010, 9:173-183.

47. Ptacek J, Devgan G, Michaud G, Zhu H, Zhu X, Fasolo J, Guo H, Jona G, Breitkreutz A, Sopko R, et al: Global analysis of protein phosphorylation in yeast. Nature 2005, 438:679-684.

48. Fasolo J, Sboner A, Sun MG, Yu H, Chen R, Sharon D, Kim PM, Gerstein M, Snyder M: Diverse protein kinase interactions identified by protein microarrays reveal novel connections between cellular processes. Genes Dev 2011, 25:767-778.

49. Thomas BJ, Rothstein R: Elevated recombination rates in transcriptionally active DNA. Cell 1989, 56:619-630.

50. Görner W, Durchschlag E, Wolf J, Brown E, Ammerer G, Ruis H, Schüller C: Acute glucose starvation activates the nuclear localization signal of a stress-specific yeast transcription factor. Embo J 2002, 21:135-144.

51. De Wever V, Reiter W, Ballarini A, Ammerer G, Brocard C: A dual role for PP1 in shaping the Msn2-dependent transcriptional response to glucose starvation. Embo J 2005, 24:4115-4123.

52. Belinchón MM, Flores CL, Gancedo JM: Sampling Saccharomyces cerevisiae cells by rapid filtration improves the yield of mRNAs. FEMS Yeast Research 2004, 4:751-756.

53. Daran-Lapujade P, Jansen ML, Daran JM, van Gulik W, de Winde JH, Pronk JT: Role of transcriptional regulation in controlling fluxes in central carbon metabolism of Saccharomyces cerevisiae. A chemostat culture study. J Biol Chem 2004, 279:9125-9138.

54. De Nicola R, Hazelwood L, De Hulster E, Walsh M, Knijnenburg T, Reinders M, Walker G, Pronk J, Daran J, Daran-Lapujade P: Physiological and transcriptional responses of Saccharomyces cerevisiae to zinc limitation in chemostat cultures. App/ Environ Microbiol 2007, 73:7680-7692.

55. Tusher V, Tibshirani R, Chu G: Significance analysis of microarrays applied to the ionizing radiation response. Proc Natl Acad Sci USA 2001, 98:5116-5121.

doi:10.1186/1471-2164-12-405

Cite this article as: Livas et al:: Transcriptional responses to glucose in Saccharomyces cerevisiae strains lacking a functional protein kinase A. BMC Genomics 2011 12:405.

\section{Submit your next manuscript to BioMed Central and take full advantage of:}

- Convenient online submission

- Thorough peer review

- No space constraints or color figure charges

- Immediate publication on acceptance

- Inclusion in PubMed, CAS, Scopus and Google Scholar

- Research which is freely available for redistribution

Submit your manuscript at www.biomedcentral.com/submit
Ciomed Central 